\title{
BMJ Open Multidrug and optimal heart failure therapy prescribing in older general practice populations: a clinical data linkage study
}

\author{
Claire A Rushton, ${ }^{1}$ Anna Strömberg, ${ }^{2}$ Tiny Jaarsma, ${ }^{3}$ Umesh T Kadam ${ }^{4}$
}

To cite: Rushton $\mathrm{CA}$, Strömberg A, Jaarsma T, et al. Multidrug and optimal heart failure therapy prescribing in older general practice populations: a clinical data linkage study. BMJ Open 2014:4:e003698. doi:10.1136/bmjopen-2013003698

- Prepublication history for this paper is available online. To view these files please visit the journal online (http://dx.doi.org/10.1136/ bmjopen-2013-003698).

Received 1 August 2013 Revised 15 November 2013 Accepted 22 November 2013

CrossMark

For numbered affiliations see end of article.

Correspondence to

Claire A Rushton;

c.a.rushton@keele.ac.uk

\section{ABSTRACT}

Objective: To investigate multidrug therapy in the cardiovascular disease (CVD) population and whether it was associated with suboptimal drug prescribing in heart failure (HF).

Design: A population-based cross-sectional clinical data linkage study.

Setting: The clinical database populations were registered with three general practices in North Staffordshire that are part of a research network.

Participants: 3155 patients aged 50 years and over were selected on the basis of a CVD-related prescription and a CVD consultation code applied to their electronic medical record in a 2-year time period. All available diagnostic data were linked to all drugs prescribed data during this time period. Two study groups were: (1) HF and (2) non-HF CVD (reference group).

Exposure: A standard drug formulary system was used to define four multidrug count categories based on the number of different British National Formulary drug chapters prescribed at the same time.

Primary and secondary outcome measures: Optimal HF therapy was defined as the prescribing of ACE inhibitor (ACEi) or a combination of ACEi and $\beta$ blocker in the 2-year time window. An additional three specific CVD drug categories that are indicated in $\mathrm{HF}$ were also measured.

Results: The HF group, compared with the reference group, had higher non-CVD multidrug therapy ( $26 \%$ with 7 or more counts compared with $14 \%$ in the nonHF CVD reference group). For the first-choice optimal drug treatment for HF with ACEi (64\%) or ACEi and $\beta$ blocker combined therapy (23\%), the multidrugadjusted associations between the $\mathrm{HF}$ group and the reference group were OR $3.89 ; 95 \% \mathrm{Cl} 2.8$ to 5.5 and $1.99 ; 1.4$ to 2.9 , respectively. These estimates were not influenced by adjustment for sociodemographic factors and multidrug counts.

Conclusions: Multidrug therapy prescribing is much higher in the HF group than in a comparable CVD group but did not influence optimal drug prescribing.

\section{BACKGROUND}

The prevalence of heart failure (HF) is increasing in older populations ${ }^{1}$ and drug

\section{Strengths and limitations of this study}

- The study has investigated multidrug therapy in patients with heart failure (HF) drawn from a large cardiovascular population. The general practice setting is where most of the patients are seen and followed-up, and where most of the drugs are prescribed on a long-term basis. The cardiovascular group provided an appropriate comparison for investigating multidrug therapy and the use of common cardiovascular drugs such as $\beta$-blockers and ACE inhibitor in HF. Accuracy of recording of morbidity and prescription data in the UK general practice has been found to range from moderate to excellent. ${ }^{42}$ This study selected patients on the basis of cardiovascular disease (CVD) prescriptions which are known to be more accurately and consistently recorded ${ }^{43}$ and this was linked to consultation data. Furthermore, the general practices used for this study were all part of the North Staffordshire Primary Care Research Consortium which is a network for supporting good-quality epidemiological data. However, the diagnostic categories do not convey the other aspect of measurement, which is severity of $\mathrm{HF}$, which may influence the levels of prescribing.

- The study used recorded data from 2000 to 2001 and the use of ACEi and $\beta$-blocker combination in HF has increased since this time period. ${ }^{16}$ So part of the explanations for the low prescribing of combination therapy could be that there is often a time lag between the current best evidence and use in practice. However, the data provided an appropriate basis for investigating the a priori hypothesis that multidrug prescription influences optimal HF drug prescribing and we were able to test this on emerging and established definitions of optimal therapy.

- Multidrug therapy in this study has been defined by chapter counts in order to characterise more clearly the scope of non-CVD multidrug therapy in this group. While this is one epidemiological perspective, there is still scope for more refined future definitions that take account of dose and duration of prescribed drugs. 
therapy is the key therapeutic approach to manage symptoms and reduce mortality, ${ }^{2}$ yet mortality, morbidity and healthcare costs remain high. ${ }^{3}{ }^{4}$ Despite strong evidence on the efficacy of specific cardiovascular drugs in improving HF outcomes, the underuse of evidencebased drug therapy persists. ${ }^{5-7}$ A key challenge in optimal prescribing is the multiple numbers of other diseases and associated drugs that patients with HF often experience. $^{8}$

Older adults are exposed to an increased number of drug prescriptions and the associated consequence of potentially inappropriate prescribing. ${ }^{9}$ This is exacerbated in $\mathrm{HF}$ where higher levels of comorbidity ${ }^{10}$ are associated with an increased number of drugs used in each patient. ${ }^{11}$ In HF, however, there are a number of non-cardiovascular disease (CVD) drugs that are contraindicated ${ }^{12} 13$ and $17 \%$ of patients with HF have reported adverse effects from their multiple drug regimens. ${ }^{14} 15$ A previous research study showed that comorbidity strongly influenced the prescribing of recommended drug regimens in HF but $44 \%$ of patients' suboptimal drug regimens were unexplained by comorbidity. ${ }^{16}$ One suggestion is that a patient's multidrug therapy may result in suboptimal drug prescribing due to the clinical problem of drug interactions or adverse effects. ${ }^{17}$

Few studies have characterised the drug regimens in HF compared with other CVD populations or how multidrug therapy affects the prescribing of recommended CVD drugs in the HF group compared with other non-HF CVD groups. The aims of the study were (1) to describe multidrug prescribing in the cardiovascular general practice population and then (2) to test the hypothesis that non-CVD multidrug prescribing is associated with suboptimal HF drug therapy.

\section{METHODS}

Design

This study used a large electronic medical database linking diagnostic data to all prescribed drugs covering a 2-year time period. Within each study general practice, diagnostic data and prescribed data were extracted from the medical records and linked using individual patient National Health Service (NHS) identifiers. The linked data were then anonymised prior to the data analysis. The clinical database populations were registered with one of the three general practices that are part of a General Practice Research Network. Registered patients with these three practices had participated in a population survey in $2001,{ }^{18}$ and a confidential study database was created which had ethics approval for research.

\section{Study population}

The study population was aged 50 years and older and was from an urban area of England that has a higher than average number of people with health deprivation and disability. Ninety-eight per cent of Staffordshire is Caucasian. ${ }^{19}$ The three general practices routinely computer code clinical encounters using the Read code classification $^{20}$ and drug prescriptions using the British National Formulary (BNF). ${ }^{21}$ The Read code classification has main chapters on symptoms and diagnostic conditions, and within the main chapter there are four subhierarchical levels. The BNF has 15 main drug chapters, each related to a different body system, which is then divided into different subclassifications of drugs within the same chapter. Both classifications provide a standard coding framework within which diagnostic and pharmacoepidemiology studies can be investigated in general practice populations.

From the overall study database, we identified a study population of 3155 patients who had received at least one CVD drug prescription (BNF Chapter 2-'cardiovascular system') and one CVD-related consultation code in the study-defined time period (2000-2001). The data retrieved from the database also included the patient's age, gender and a measure of deprivation. For the measure of deprivation, we used the Index of Multiple Deprivation (IMD) score which is a nationally recognised measure of deprivation at the small area level. ${ }^{22}$

\section{Study groups}

Two study groups were constructed on the basis of the linked diagnostic and prescribed drug information for the total cardiovascular population of 3155 patients during the 2-year time window of observation. The groups were based on CVD diagnostic data, comprising the HF group and a non-HF CVD group. Patients with CVD were identified by the Read code ' $G$ ' prefix applied on their clinical record. The HF group was identified by a range of codes with the prefix 'G58' which relates to different HF diagnostic categories, for example, congestive HF, decompensated HF or left ventricular failure. This group was separated from the broader cardiovascular group.

\section{Definition of multidrug therapy}

There are no standard approaches to define multidrug therapy. Previous studies have used the number of prescribed drugs to describe polypharmacy with various cut-off points defined. ${ }^{23}{ }^{24}$ While this approach may be a useful indication of the level of prescribing in older adults it is limited in its representation of the range of different drugs that a patient with multimorbidity may be exposed to. One method that has been developed in our previous work, ${ }^{25}$ and applied in this study summarises the counts of the 15 different system-related drugs that a person can be prescribed based on the BNF classification. Multidrug is defined as two or more main drug groups prescribed at the same time. This definition provides a proxy measure of the range or spectrum of multidrug prescribing. Since the main BNF chapter for CVD was used to select the study population, the other 14 main BNF chapters provided the basis for counting the number of non-CVD drug groups that a patient had been prescribed in the 2-year time-window. This 
multidrug measure relates to at least one prescription for one of the main BNF chapters, and does not include repeated prescriptions under the same chapter. The non-CVD drug counts were then categorised into four count categories: (1) 0 (ie, no non-CVD drugs), (2) 1-3, (3) 4-6 and (4) 7 or more.

\section{Definition of CVD drug measures}

There are a wide range of CVD drugs used in HF, with some that are recommended in all patients and others that are indicated depending on the clinical context. Using the American and European national guidance for $\mathrm{HF}^{2}{ }^{26}$ four CVD drug groups were constructed (A-D) which represent the potential CVD drugs that might be prescribed for HF. Category A included the drugs recommended for all patients who do not have a clinical contraindication and was subdivided into two study definitions of 'optimal' HF drug therapy. Groups B-D included the drugs that are indicated depending on the clinical context. The four drug groups were defined as follows:

1. Group A, 'optimal' therapy, was defined by (1) the combined prescribing of ACE inhibitor (ACEi) and $\beta$-blockers; national guidelines recommend the use of both these CVD drugs as the first-choice treatment for HF with left ventricular systolic dysfunction which forms the largest part of diagnosed $\mathrm{HF}^{2}$ and (2) the prescription of at least ACEi over the 2-year study period. As the evidence on ACEi and $\beta$-blocker combination therapy was still being established within the clinical guidelines at the time of the study inclusion, ${ }^{27} 28$ the second definition was created to reflect the established practice at that time. Not all patients with $\mathrm{HF}$ are able to tolerate these drugs, and substitution by group B drugs may be required, but we wanted to test the a priori hypothesis that multidrug therapy influences the prescription of this recommended 'first-choice' therapy. Both drugs are also used more widely in the management of ischaemic heart disease and hypertension, which may be separate to or coexist in patients with HF.

2. Group B includes aldosterone antagonists, angiotensin-11 receptor antagonists and the vasodilator combination, hydralazine and nitrate. ${ }^{29}{ }^{30}$ These drugs are used as an alternative first-line treatment in patients who are intolerant of ACEi or as second-line treatment in patients who remain symptomatic on first-line treatment using group A drugs.

3. Group $\mathrm{C}$ includes Digoxin which is recommended for symptom reduction ${ }^{31}$ in patients who remain symptomatic following prescription of group A and B drugs as well as for patients with $\mathrm{HF}$ with atrial fibrillation.

4. Group D includes diuretics that are used in all patients with $\mathrm{HF}$ periodically to treat symptoms related to fluid retention. ${ }^{32}$ The diuretics group excluded aldosterone antagonists (spironolactone and eplerenone) which were classified in group B.

\section{Statistical analysis}

Age of the study population was categorised into four age bands and the IMD score was categorised into four quartiles (quartiles 1 (least deprived) to 4 (most deprived)). There were two stages to the analyses.

First, descriptive data for the study groups and non-CVD multidrug therapy are presented. The two study groups are described by age bands, gender and deprivation quartiles and then non-CVD multidrug prescribing is described for the overall study population by these study factors and separately for the two study groups.

Second, the adjusted associations between the HF group compared with the non-HF CVD group and the study CVD drug measures are presented. Using logistic regression methods with $95 \%$ CIs, the associations between the HF group and the four CVD study drug measures compared with the non-HF CVD reference group were estimated. OR estimates were adjusted first for age, gender and deprivation quartiles. Next, adjustment was made for non-CVD multidrug counts. This was first conducted by count category and then as a continuous variable. These steps of adjustment were performed so that the influence of non-CVD multidrug therapy on the observed associations could be identified.

\section{RESULTS}

\section{Study population}

Of the 3155 study patients, $170(5.4 \%)$ patients were in the HF group and $2985(94.6 \%)$ were in the reference group. The HF group was older than the other group. Forty-four per cent of the HF group was in the oldest age category compared with $16 \%$ of the non-HF CVD group. Only 3\% of the HF group was in the youngest age category. There were slightly less women than men in the HF group (48\%) but more women than men in the non-HF CVD group (54\%). There was a higher proportion of the HF group with an affluent status (29\%) than the most deprived status (19\%; table 1$)$.

\section{Multidrug therapy prescribing}

Older age groups had a higher number of multidrug counts, with $67 \%$ of the population aged 80 years and over being prescribed four or more non-CVD drug groups, compared with $42 \%$ of the population aged 50 59 years. Women had higher multidrug counts than men, with $62 \%$ being prescribed four or more non-CVD drug groups compared with just under half of the men. The most deprived quartile had higher multidrug counts of 4 or more non-CVD groups than the least deprived quartile ( $65 \%$ vs $48 \%$, respectively). The HF group had higher counts of multidrug therapy than the other CVD group. A quarter $(26 \%)$ of the HF group had 7 or more multidrug counts compared with $14 \%$ of the non-HF CVD reference group (table 2). 
Table 1 Study population characteristics

\begin{tabular}{|c|c|c|c|}
\hline \multirow[b]{2}{*}{ Factor } & \multirow[b]{2}{*}{ Categories } & \multicolumn{2}{|l|}{ CVD groups } \\
\hline & & $\begin{array}{l}\text { Non-HF CVD (reference) } \\
(\mathrm{n}=2985)\end{array}$ & HF $(n=170)$ \\
\hline \multirow[t]{5}{*}{ Age } & $50-59$ & $534(17.9)$ & $5(2.9)$ \\
\hline & $60-69$ & $954(32)$ & $30(17.6)$ \\
\hline & 70-79 & $1034(34.6)$ & 61 (35.9) \\
\hline & $80>$ & $463(15.5)$ & $74(43.5$ \\
\hline & Mean age (SD) & $69.5(9.5)$ & $77.0(8.2)$ \\
\hline Gender & Women & $1623(54.4)$ & $82(48.2)$ \\
\hline \multirow[t]{4}{*}{ Deprivation quartiles* } & Q1-least deprived & 714 (23.9) & $49(28.8)$ \\
\hline & Q2 & $666(22.3)$ & $45(26.5)$ \\
\hline & Q3 & $782(26.2$ & $44(25.9)$ \\
\hline & Q4-most deprived & $822(27.5)$ & $32(18.8)$ \\
\hline
\end{tabular}

\section{Associations between study groups and CVD drugs}

More patients with HF were prescribed each of the CVD drug categories than the reference group. In the HF group, the most frequent drug therapy prescribed was diuretics $(95 \%)$, with $28 \%$ for digoxin and $20 \%$ for the antagonist and vasodilator group (table 3 ). Of the HF group, $64 \%$ were on at least ACEi and $24 \%$ were on combined therapy. After adjustment for age, gender and deprivation, there were significant associations between the HF group and the prescribing of all CVD drug groups, but the strength of the associations varied. The strongest associations between the HF group and drug therapy compared with the reference group were with diuretics (OR 14.4; 95\% CI 7.0 to 29.6). The adjusted estimate for the association between the HF group and at least ACEi was 3.99; 95\% CI 2.9 to 5.6, and for combined therapy compared with the reference group was 1.98; 95\% CI 1.4 to 2.9. Additional adjustment for non-CVD multidrug therapy counts did not alter the associations between HF and any of the related drug groups. Comparing the HF group with the reference group for optimal therapy, the multidrug-adjusted associations for at least ACEi were: HF group-3.89 (2.8 to 5.5) and for combined therapy were: HF group-OR 1.99 (95\% CI 1.4

Table 2 Cardiovascular study population and multidrug therapy

\begin{tabular}{|c|c|c|c|c|c|c|}
\hline \multirow[b]{2}{*}{ Factor } & \multirow[b]{2}{*}{ Categories } & \multirow[b]{2}{*}{ Mean } & \multicolumn{4}{|c|}{ Non-CVD multidrug counts*† } \\
\hline & & & $\overline{0}$ & $1-3$ & $4-6$ & 7 or more \\
\hline Total study population & & & $162(5.1)$ & $1220(38.7)$ & $1316(41.7)$ & $457(14.5)$ \\
\hline \multirow[t]{4}{*}{ Age } & $50-59$ & 4.2 & $54(10)$ & $257(47.2)$ & $187(34.7)$ & $41(7.6)$ \\
\hline & $60-69$ & 4.8 & $62(6.3)$ & $397(40.3)$ & 397 (40.3) & $128(13)$ \\
\hline & $70-79$ & 5.2 & $31(2.8)$ & $404(36.9)$ & $483(44.1)$ & $177(16.2)$ \\
\hline & $80 \geq$ & 5.6 & $15(2.8)$ & $162(30.2)$ & $249(46.4)$ & $111(20.7)$ \\
\hline \multirow[t]{2}{*}{ Gender } & Female & 5.3 & $58(3.4)$ & $594(34.8)$ & $757(44.4)$ & $296(17.4)$ \\
\hline & Male & 4.6 & $104(7.2)$ & $626(43.2)$ & $559(38.6)$ & $161(11.1)$ \\
\hline \multirow[t]{4}{*}{ Deprivation quartiles $†$} & Q1-least deprived & 4.6 & $43(5.6)$ & $351(46)$ & 289 (37.9) & $80(10.5)$ \\
\hline & Q2 & 4.9 & $45(6.3)$ & $273(38.4)$ & $294(41.4)$ & 99 (13.9) \\
\hline & Q3 & 5.0 & $45(5.4)$ & 321 (38.9) & 346 (41.9) & $114(13.8)$ \\
\hline & Q4-most deprived & 5.5 & $29(3.4)$ & $274(32.1)$ & 387 (45.3) & $164(19.2)$ \\
\hline \multirow[t]{2}{*}{ CVD groups } & non-HF CVD & 4.9 & $161(5.4)$ & $1176(39.4)$ & $1235(41.4)$ & $413(13.8)$ \\
\hline & $\mathrm{HF}$ & 6.1 & $1(.6)$ & $44(25.9)$ & $81(47.6)$ & $44(25.9)$ \\
\hline
\end{tabular}

Figures in brackets are the percentage of each drug count category within each factor subgroup.

${ }^{*}$ Non-CVD multidrug counts = number of additional chapters (from 14 possible BNF chapters) from which at least one drug was prescribed over a 2-year time period (2000-2001).

Both CVD groups are defined by patients who received a British National Formulary Chapter 2 (CVD) drug prescription over a 2-year time period. Two mutually exclusive groups in the 2-year time-period (1) reference group = CVD drug and CVD diagnostic categories (other than HF) in the record review (2) HF group = CVD drugs and HF diagnostic categories.

$\dagger(n=3154)$.

BNF, British National Formulary; CVD, cardiovascular disease; HF, heart failure. 
Table 3 Associations between cardiovascular groups and heart failure-related drugs

\begin{tabular}{|c|c|c|c|c|c|}
\hline \multirow[b]{2}{*}{$\begin{array}{l}\text { Study groups } \\
\text { by drug } \\
\text { outcomes }\end{array}$} & \multirow[b]{2}{*}{$\begin{array}{l}\text { Not } \\
\text { prescribed n (\%) }\end{array}$} & \multirow[b]{2}{*}{$\begin{array}{l}\text { Prescribed } \\
\text { n (\%) }\end{array}$} & \multirow[b]{2}{*}{$\begin{array}{l}\text { Unadjusted } \\
\text { OR }(95 \% \mathrm{Cl})\end{array}$} & \multicolumn{2}{|l|}{ Adjusted OR (95\% Cl) } \\
\hline & & & & $\begin{array}{l}\text { Age, gender and } \\
\text { deprivation status }\end{array}$ & $\begin{array}{l}\text { Age, gender, deprivation } \\
\text { status* and non-CVD } \\
\text { multidrug counts }\end{array}$ \\
\hline \multicolumn{6}{|l|}{ Group A } \\
\hline \multicolumn{6}{|l|}{ At least ACEi } \\
\hline Reference & $1971(66)$ & $1014(34)$ & 1.0 & 1.0 & 1.0 \\
\hline $\mathrm{HF}$ & $62(36.5)$ & $108(63.5)$ & 3.39 (2.5 to 4.7$)$ & 3.99 (2.9 to 5.6$)$ & 3.89 (2.8 to 5.5$)$ \\
\hline \multicolumn{6}{|c|}{ ACEi and $\beta$ - blocker combined } \\
\hline Reference & $2536(85.0)$ & $449(15.0)$ & 1.0 & 1.0 & 1.0 \\
\hline HF & $131(77.1)$ & 39 (22.9) & $1.68(1.2$ to 2.4$)$ & 1.98 (1.4 to 2.9$)$ & 1.99 (1.4 to 2.9$)$ \\
\hline \multicolumn{6}{|l|}{ Group B } \\
\hline Reference & $2823(94.6)$ & $162(5.4)$ & 1.0 & 1.0 & 1.0 \\
\hline HF & $136(80.0)$ & $34(20.0)$ & 4.36 (2.9 to 6.6$)$ & 5.06 (3.3 to 7.8$)$ & 4.61 (3.0 to 7.1$)$ \\
\hline \multicolumn{6}{|l|}{ Group C } \\
\hline Reference & $2830(94.8)$ & $155(5.2)$ & 1.0 & 1.0 & 1.0 \\
\hline HF & $123(72.4)$ & $47(27.6)$ & $6.98(4.8$ to 10.1$)$ & 4.88 (3.3 to 7.2$)$ & 4.75 (3.2 to 7.0$) 0$ \\
\hline \multicolumn{6}{|l|}{ Group D } \\
\hline Reference & $1265(42.4)$ & $1720(57.6)$ & 1.0 & 1.0 & 1.0 \\
\hline HF & $8(4.7)$ & $162(95.3)$ & 14.89 (7.3 to 30.4 & 14.7 (7.2 to 30.2$)$ & 14.4 (7.0 to 29.6$)$ \\
\hline \multicolumn{6}{|c|}{$\begin{array}{l}\text { Both CVD groups are defined by patients who received a BNF Chapter } 2 \text { (CVD) drug prescription over a 2-year time period. Two mutually } \\
\text { exclusive groups in the } 2 \text {-year time period (1)reference group=CVD drug and CVD diagnostic categories (other than HF) in the record review } \\
\text { (2) HF group=CVD drugs and HF diagnostic categories. Heart failure drug groups=groups of HF drugs which at least one drug was prescribed } \\
\text { over a 2-year period; } A \text { (optimal therapy)=(a) at least ACEi and (b) ACEi and } \beta \text {-blocker combined, } B=\text { =aldosterone antagonists, angiotensin II } \\
\text { receptor antagonists or hydralazine/nitrate combination, } C=\text { digoxin, } D=\text { diuretics. } \\
\text { *(n=3154). } \\
\text { BNF, British National Formulary; CVD, cardiovascular disease; HF, heart failure. }\end{array}$} \\
\hline
\end{tabular}

to 2.9; table 3). Adjustment of multidrug counts as a continuous variable made no difference to the associations between the HF group and both optimal HF drug definitions compared with the reference group-at least ACEi were: HF group-3.89 (2.8 to 5.5) and combined therapy were: HF group 2.02 (1.4 to 3.0 ).

\section{DISCUSSION}

In a large cardiovascular general practice population, our study showed that multidrug prescribing was common, but did not influence the association between the HF group and the prescription of optimal therapy. The older HF group had the highest non-CVD multidrug therapy, yet was still much more likely to receive cardiovascular drugs than the non-HF CVD group, which also has other indications for use in general practice such as hypertension and ischaemic heart disease.

We found that two-thirds of the HF population were prescribed at least ACEi and a quarter of the HF population were prescribed ACEi and $\beta$-blockers combined. A European study found the presence of comorbidity to account for some but not all of the non-adherence to the guideline-recommended drug prescribing. ${ }^{16}$ The prescribing of high amounts of multiple drugs found in HF populations may explain the suboptimal prescribing of $\mathrm{HF}$ drug therapy due to contraindications and adverse effects, but this was found not to be the case in this study. An additional finding in the patients with HF was the higher relative prescribing of diuretics compared with the other CVD drug groups. Diuretics are used to control HF symptoms related to fluid retention, such as breathlessness, but their impact on outcomes such as mortality is unclear with some studies showing adverse effects. $^{32}$ The aim of HF medical treatment is to optimise the mainline drug therapies such as ACEi and $\beta$-blockade and only use diuretics intermittently for symptom relief. The higher relative prescribing of diuretics found in this study potentially represents the traditional drug therapy approach in HF up until the early 1990s when diuretics were the main stay of treatment. ${ }^{33}$

Studies have shown that the number of drugs that a patient is prescribed at any one time increases with age and female gender. ${ }^{34}$ Our study findings demonstrate that this was reflected within the general CVD population in terms of other multiple drug therapies. Comparable studies have shown that the average age, the number of medications and the number of comorbidities to be high and increasing in the HF populations. $^{10} 35$ Our findings also highlight the increased multidrug prescribing in the most deprived cardiovascular populations.

Adherence to evidence-based guidelines for optimal HF drug therapy by clinicians is poor $^{36}$ and this adherence is lower in general practice settings than specialist settings. ${ }^{37}$ Our findings found that most patients with HF were on at least ACEi and a quarter of patients with HF were prescribed ACEi and $\beta$-blockers in combination. Qualitative studies investigating the clinical reasons for non-adherence to guidelines include the side effects of 
drugs in patients with HF with comorbidity and multidrug therapy, ${ }^{38} 39$ but our study findings suggest that the number and range of non-CVD multidrugs did not influence the prescribing of HF combination drug therapy.

We defined optimal HF prescribing in this study as the prescription of recommended first-line drug treatments. Broader definitions of 'suboptimal' therapy include the 'over-use' of drugs which expose patients to the risks associated with polypharmacy and the inappropriate use' of drug therapies. ${ }^{9}$ Older people with multimorbidity are particularly vulnerable to suboptimal prescribing and one study found over one-third of people aged 70 years or older to be prescribed at least one potentially inappropriate prescription. This risk increases with the number of drugs prescribed. ${ }^{40}$ Our study demonstrates that older people with HF are exposed to high levels of non-CVD multidrug counts. While this did not influence the prescription of HF first-line treatment it has important clinical implications for the safety and appropriateness of drug regimens for the older patient with HF.

\section{IMPLICATIONS}

This study provides population-based evidence on the multiple drug therapies that patients with $\mathrm{HF}$ are exposed to and the influence on the prescribing of specific HF drug therapy. While multidrug therapy was not associated with HF prescribing, there is clear scope for improvements in prescribing within the CVD population. Multidrug prescribing and the scale of prescribing were high in this study and expose patients to potential adverse effects and inappropriate drug prescribing. Regular review of drug regimens for patients with $\mathrm{HF}$ is required with particular attention to older age groups, women and the most deprived populations. Other studies have found that patients who are prescribed appropriate drugs for $\mathrm{HF}$ often receive suboptimal dosing, ${ }^{37} 3941$ but the influence of multidrug therapy on this issue is unclear. Further work is still needed to investigate the impact of multidrug therapy on optimal therapy in terms of dose and duration of therapy, and whether multidrug therapy influences the prognostic outcomes in conditions such as HF.

\section{Author affiliations \\ ${ }^{1}$ School of Nursing and Midwifery, Keele University, Stoke-on-Trent, UK \\ ${ }^{2}$ Department of Medical and Health Sciences, Linköping University and Department of Cardiology, County Council of Östergötland, Linkoping, Sweden \\ ${ }^{3}$ Department of Social and Welfare Studies, Linköping University, Linkoping, Sweden \\ ${ }^{4}$ Health Services Research Unit, Keele University, Newcastle-under-Lyme, UK}

Acknowledgements The authors would like to thank the patients and the general practices who participated in the study.

Contributors CAR was the lead author for this paper and involved in all stages outlined below.AS and TJ contributed to the drafting of the manuscript, critical revision of the manuscript and supervision. UTK was the lead supervisor for CAR and in addition to contributing to the drafting and critical revision of the manuscript, also contributed to the conception and design of the research, acquisition of data, analysis and interpretation of the data and statistical analysis.

Funding The funding was by the Florence Nightingale Foundation.

Competing interests CAR was supported by a Florence Nightingale Foundation grant, Keele Nursing School Link Fellowship, and currently by a National Institute for Health Research (NIHR) Doctoral Research Fellowship.

Ethics approval North Staffordshire Local Research Ethics Committee.

Provenance and peer review Not commissioned; externally peer reviewed.

Data sharing statement No additional data are available.

Open Access This is an Open Access article distributed in accordance with the Creative Commons Attribution Non Commercial (CC BY-NC 3.0) license, which permits others to distribute, remix, adapt, build upon this work noncommercially, and license their derivative works on different terms, provided the original work is properly cited and the use is non-commercial. See: http:// creativecommons.org/licenses/by-nc/3.0/

\section{REFERENCES}

1. Thomas S, Rich M. Epidemiology, pathophysiology, and prognosis of heart failure in the elderly. Clin Geriatr Med 2007;23:1-10.

2. Lindenfeld J, Albert N, Boehmer J, et al. HFSA 2010 comprehensive heart failure practice guideline. J Card Fail 2010;16:e1-194.

3. NICE. Chronic heart failure: National Clinical Guideline for diagnosis and management in primary and secondary care. London: Royal College of Physicians, 2010.

4. Stewart S, Jenkins A, Buchanc S, et al. The current cost of heart failure to the National Health Service in the UK. Eur J Heart Fail 2002;4:361-71.

5. The NHS Information Centre. The national heart failure audit. Leeds: NHS, Information Centre for Health and Social Care, 2010.

6. Ceiaa F, Fonsecab C, Motad T, et al. Aetiology, comorbidity and drug therapy of chronic heart failure in the real world: the EPICA substudy. Eur J Heart Fail 2004;6:801-6.

7. Gambassi G, Forman D, Lapane K, et al. Management of heart failure among very old persons living in long-term care: has the voice of trials spread? Am Heart $J$ 2000;139:85-93.

8. Lindenfeld J. The comorbidity conundrum: a focus on the role of noncardiovascular chronic conditions in the heart failure patient. Curr Cardiol Rep 2012;14:276-84.

9. Nyborg G, Straand J, Brekke M. Inappropriate prescribing for the elderly-a modern epidemic? Eur J Clin Pharmacol 2012;68:1085-94.

10. Wong CY, Chaudhry SI, Desai MM, et al. Trends in comorbidity, disability, and polypharmacy in heart failure. $\mathrm{Am} J \mathrm{Med}$ 2011;124:136-43.

11. Masoudi F, Baillie C, Wang Y, et al. The complexity and cost of drug regimens of older patients hospitalized with heart failure in the United States, 1998-2001. Arch Intern Med 2005;165:2069-76.

12. Amabile CM, Spencer AP. Keeping your patient with heart failure safe: a review of potentially dangerous medications. Arch Intern Med 2004;164:709-20.

13. Rushton CA, Kadam UT. Polypharmacy in heart failure: a growing challenge. Br J Card Nurs 2011;6:214-20.

14. De Smedt RHE, Jaarsma T, Haaijer-Ruskamp FM, et al. The impact of perceived adverse effects on medication changes in heart failure patients. J Card Fail 2010;16:135-41.

15. Gandhi T, Weingart S, Borus J, et al. Adverse drug events in ambulatory care. N Engl J Med 2003;348:1556-64.

16. Sturm H, Haaijer-Ruskamp F, Veeger N, et al. The relevance of comorbidities for heart failure treatment in primary care: a European Survey. Eur J Heart Fail 2006;8:31-7.

17. Dahlstrom U. Frequent non-cardiac comorbidities in patients with chronic heart failure. Eur J Heart Fail 2005;7:309-16.

18. Thomas E, Wilkie R, Peat G, et al. The North Staffordshire Osteoarthritis Project-NorStOP: prospective, 3-year study of the epidemiology and management of clinical osteoarthritis in a general population of older adults. BMC Musculoskelet Disord 2004;5:2. http://www.biomedcentral.com/content/pdf/1471-2474-5-2.pdf (accessed Sep 2013)

19. Trinder P. South Stoke Primary Care Trust. Demography and Deprivation Profile. Directorate of Health Policy and Public Health, North Staffordshire Health, 2002 http://www.nsha.co.uk/home/library/hp/hp

20. Chisholm J. The Read clinical classification. BMJ 1990;300:1092

21. British Medical Association. Royal Pharmaceutical Society of Great Britain. British National Formulary, No. 58. London: BMJ Group and Pharmaceutical Press, September 2009. 
22. Office of the Deputy Prime Minister. The English Indices of deprivation 2004-summary (revised); http://www.brent.gov.uk/tps.nsf/ Files/LBBA869/\$FILE/sd62blndicesofDeprivation2004.pdf

23. Gnjidic D, Hilmer S, Blyth F, et al. Polypharmacy cutoff and outcomes: five or more medicines were used to identify community-dwelling older men at risk of different adverse outcomes. J Clin Epidemiol 2012;65:989-95.

24. Lai S, Liao K. Optimal cutoff of polypharmacy and outcomes. J Clin Epidemiol 2013;66:465.

25. Kadam U. Potential health impacts of multiple drug prescribing for older people: a case-control study. Br J Gen Pract 2011;61:128-30.

26. McMurray J, Adamopoulos S, Anker S, et al. ESC guidelines for the diagnosis and treatment of acute and chronic heart failure 2012. Oxford University Press, 2012.

27. HFSA. Heart Failure Society of America (HFSA) practice guidelines. HFSA guidelines for management of patients with heart failure caused by left ventricular systolic dysfunction-pharmacological approaches. J Card Fail 1999;5:357-82.

28. Remme W, Swedburg K. Guidelines for the diagnosis and treatment of chronic heart failure. Task Force for the Diagnosis and Treatment of Chronic Heart Failure, European Society of Cardiology. Eur Heart J 2001;22:1527-60.

29. McMurray J, Östergren J, Swedberg K, et al. Effects of candesartan in patients with chronic heart failure and reduced left-ventricular systolic function taking angiotensin-converting-enzyme inhibitors: the CHARM-Added trial. Lancet 2003;362:767-71.

30. Pitt B, Zannad F, Remme WJ, et al. The effect of spironolactone on morbidity and mortality in patients with severe heart failure. $N$ Engl $J$ Med 1999;341:709-17.

31. The Digitalis Investigation Group. The effect of digoxin on mortality and morbidity in patients with heart failure. $N \mathrm{Engl} J \mathrm{Med}$ 1997;336:525-33.

32. Domanski M, Norman J, Pitt B, et al. Diuretic use, progressive heart failure, and death in patients in the Studies of Left Ventricular Dysfunction (SOLVD). J Am Coll Cardiol 2003;42:705-8.

33. Cowley AJ, Stainer K, Wynne RD, et al. Symptomatic assessment of patients with heart failure: double-blind comparison of increasing doses of diuretics and captopril in moderate heart failure. Lancet 1986;2:770-2.

34. Viktil K, Blix H, Moger $\mathrm{T}$, et al. Polypharmacy as commonly defined is an indicator of limited value in the assessment of drug-related problems. Br J Clin Pharmacol 2006;63:187-95

35. Braunstein J, Anderson G, Gerstenblith G, et al. Noncardiac comorbidity increases preventable hospitalizations and mortality among medicare beneficiaries with chronic heart failure. J Am Coll Cardiol 2003;42:1226-33.

36. Komajda M, Lapuerta $\mathrm{P}$, Hermans $\mathrm{N}$, et al. Adherence to guidelines is a predictor of outcome in chronic heart failure: the MAHLER survey. Euro Heart J 2005;26:1653-9.

37. Ledwidge M, Travers B, Ryder M, et al. Specialist care of heart failure improves appropriate pharmacotherapy at the expense of greater polypharmacy and drug-interactions. Eur $J$ Heart Fail 2004;6:235-43.

38. Fuat A, Hungin A, Murphy J. Barriers to accurate diagnosis and effective management of heart failure in primary care: qualitative study. BMJ 2003;326:196-200.

39. Khunti K, Hearnshaw $\mathrm{H}$, Baker $\mathrm{R}$, et al. Heart failure in primary care: qualitative study of current management and perceived obstacles to evidence-based diagnosis and management by general practitioners. Eur J Heart Fail 2002;4:771-7.

40. Brekke M, Rognstad S, Straand J, et al. Pharmacologically inappropriate prescriptions for elderly patients in general practice: how common? Baseline data from The Prescription Peer Academic Detailing (Rx-PAD) study. Scand J Prim Health Care 2008;26:80-5.

41. Ryder M, Travers B, Timmons $L$, et al. Specialist nurse supervised in-hospital titration to target dose ACE inhibitor-is it safe and feasible in a community heart failure population? Eur $J$ Cardiovasc Nurs 2003;2:183-8.

42. Jordan K, Porcheret M, Croft P. Quality of morbidity coding in general practice computerized medical records: a systematic review. Family Practice 2004;21:396-412.

43. Thiru K, Hassey A, Sullivan F. Systematic review of scope and quality of electronic patient record data in primary care. $B M J$ 2003;326:1070. 